C2006 IEEE. Personal use of this material is permitted. However, permission to reprint/republish this material for advertising or promotional purposes or for creating new collective works for resale or redistribution to servers or lists, or to reuse any copyrighted component of this work in other works must be obtained from the IEEE. 


\title{
Fuzzy Service Quality Review in Service Oriented Architectures
}

\author{
Stefan Schmidt, Robert Steele, Member, IEEE, Tharam Dillon, Fellow, IEEE, and Elizabeth Chang,
} Member, IEEE

\begin{abstract}
The benefits of Service Oriented Architectures (SOAs) start to be recognized and implemented across distributed enterprise businesses. As SOAs promise to accomplish more dynamic and collaborative e-business scenarios, we need to ensure that critical factors such as trust, credibility and quality of service $(\mathrm{Q} 0 \mathrm{~S})$ are consistently and efficiently measured in such unsupervised environments. We propose a fuzzy logic-based model for the review of service fulfilment after a business interaction and demonstrate its functionalities in an exemplary application. The outcome of our model can then be used to adjust credibility records of third party agents which delivered opinions, to update trustworthiness values in the business partner, or to communicate the trustworthiness of the business partner to interested peer agents.
\end{abstract}

\section{INTRODUCTION}

Service Oriented Architectures (SOAs) offer new possibilities to enterprise Business-to-Business (B2B) scenarios. Services in SOAs are self-contained and do not depend on the context or state of other services [1]. However, the independent nature of services in SOAs involves risks to businesses who wish to conduct transactions using autonomous agents. It is critical for these businesses to build a network of trusted agents in order to achieve their goals successfully. To achieve a better adoption of the SOA for B2B-style interactions in distributed and loosely coupled environments, it is necessary to ensure that consistent and efficient trust measurement and trust adjustment mechanisms are in place.

Intelligent agents which are designed to act on behalf of businesses in service-oriented environments need to be outfitted with mechanisms to participate in and contribute to a virtual trust network. Agents need to evaluate trust in their

Stefan Schmidt is with the Department of Computer Science at the Faculty of Information Technology, University of Technology, Sydney, PO Box 123 Broadway, NSW 2007 Australia (phone: +61 29514 4469; fax: +61 29514 1807; email: sschmidt@it.uts.edu.au)

Associate Professor Robert Steele is with the Department of Computer Science at the Faculty of Information Technology, University of Technology, Sydney, PO Box 123 Broadway, NSW 2007 Australia (phone: +6129514 7872; fax: +6129514 4535; email: rsteele@it.uts.edu.au)

Professor Tharam Dillon is Dean of Information Technology, University of Technology, Sydney, PO Box 123 Broadway, NSW 2007 Australia (phone: +6129514 1800; fax: +61229514 1810; email: tharam@it.uts.edu.au)

Professor Elizabeth Chang is with the Department of Information Systems, Curtin University of Technology, Perth, GPO Box U1987, WA 6845 Australia (phone: +61 89266 1235; fax: +61 89266 7548; email: elizabeth.chang@cbs.curtin.edu.au) potential business partners or services during the service selection process to minimize financial or other risks. This is achieved by maintaining records of previous business interactions for future references. Furthermore, agents may also query peer agents to gain valuable information about services they have little or no previous experience with. Additionally, these trustworthiness values need to be updated regularly after the completion of a business transaction in order to increase the accuracy of future trust evaluation processes.

As SOAs promise to accomplish more dynamic and collaborative e-business scenarios, we need to ensure that critical factors such as trust, credibility and quality of service (QoS) are consistently and efficiently measured in such unsupervised environments. This paper defines the notions of $\mathrm{QoS}$ as the commitment to the negotiated and legally binding business contract between the service consumer and the service provider. This model proposes a fuzzy logic extension to the CCCI-metrics introduced by Chang, Hussain and Dillon $[2,3]$ to realize a flexible, consistent and efficient QoS review methodology for SOAs.

Why use fuzzy logic to measure the QoS in SOAs? Fuzzy logic provides a natural framework to deal with uncertainty and the tolerance of imprecise data inputs. The definition of quality measurement criteria like commitment, clarity and influence involves a high degree of subjectivity and ambiguity and are, thus, represented as fuzzy variables in our proposed model. Such criteria are often used to express decisive factors for the evaluation of the quality of a service or product during human interaction.

For example, we use linguistic variables [4, 5] such as 'slow', 'medium' and 'fast' to express the 'speed' of an object. This human approach offers the opportunity to express ones' subjective or vague observations as a fuzzy membership function rather than using a crisp number. The actual membership functions for the 'speed' fuzzy variable are defined individually based on ones' subjective perception of this criterion. For example, a cyclist uses different value ranges for the 'speed' fuzzy variable as compared to a pilot.

In this paper, we take advantage of fuzzy logic capabilities [6] and extend our existing work [7] by introducing a fuzzy logic-based QoS measurement model for service oriented architectures. 


\section{Fuzzy SERvice Quality Review Model}

This section introduces a model for the review of the quality of a completed business interaction in a Service Oriented Environment. The proposed model reduces the risk of future business interactions by allowing the service consumer agent, called Trusting Agent, to review the business interaction with the service provider agent, called Trusted Agent, after its completion. Furthermore, it is desirable to rate the quality of opinions delivered by third party agents, called Recommending Agents, which delivered their opinions about the Trusted Agent to the Trusting Agents during the trust evaluation process [8]. There are certain decisive factors which the Trusting Agent needs to measure in order to reach a fair conclusion over the performance of the Trusted Agent and the Recommending Agents. This conclusion not only allows the Trusting Agent to fine-tune its trustworthiness value of the Trusted Agent for future interactions but also allows the adjustment of the credibility values maintained for each peer agent who submitted his opinion prior to the business interaction.

We need a mechanism to ensure a fair review of the business interaction between the Trusting Agent and the Trusted Agent. This mechanism measures possible discrepancies between the defined or expected quality of service as specified in the mutually agreed upon service contract and the actual delivered quality of service as a result of the business interaction. We use the methodology known as CCCI (Correlation, Commitment, Clarity, and Influence) [2] to review the business interaction with the Trusted Agent.

The central objective of this methodology is the measurement of the correlation between the service contract both agents agreed to before their business interaction (expected behaviour) and the actually delivered services during the business interaction (actual behaviour). The overall correlation measurement is performed through the assessment of three criteria which play an important role in the business interaction review process; commitment, clarity and influence.

To make the business interaction review process possible, the Trusting Agent and the Trusted Agent complete a number of steps [2]:

1. Define the context domain and obtain the domain knowledge

2. Identify the quality aspects from the knowledge domain or abstract quality aspects from the 'service level agreement' or 'contract' or 'standard'

3. Develop assessment criteria for each quality aspect (defined quality)

4. Measure the quality or trust against assessment through the proposed Fuzzy Service Quality Review model

Both, the Trusting Agent and the Trusted Agent have previously acquired, or have previously been equipped with, the necessary domain knowledge to conduct business in a certain context, such as car sales. Using this domain knowledge, they are now capable of identifying and developing quality aspects in that context. Tables 1 and 2 provide brief examples of such criteria:

TABLE I

QUALITY CRITERIA AS DEFINED BY THE TRUSTING AGENT IN THE CAR SALES CONTEXT

\begin{tabular}{|c|l|c|}
\hline $\begin{array}{c}\text { Criterion } \\
\text { name }\end{array}$ & \multicolumn{1}{|c|}{$\begin{array}{c}\text { Requirement Specification / Expected } \\
\text { Behaviour }\end{array}$} & Influence \\
\hline $\begin{array}{c}\text { Body } \\
\text { style }\end{array}$ & Sedan & 4.5 \\
\hline Color & Red & 1 \\
\hline Condition & New & 3.5 \\
\hline
\end{tabular}

TABLE II

QUALITY CRITERIA AS DEFINED BY THE TRUSTED AGENT IN THE CAR SALES CONTEXT

\begin{tabular}{|c|l|c|}
\hline $\begin{array}{c}\text { Criterion } \\
\text { name }\end{array}$ & \multicolumn{1}{|c|}{$\begin{array}{c}\text { Requirement Specification / Expected } \\
\text { Behaviour }\end{array}$} & Influence \\
\hline Payment & Credit Card or Bank Deposit & 4 \\
\hline Delivery & Within 1 week & 3 \\
\hline
\end{tabular}

Both parties specifically agree to each quality criterion and this then is included in the negotiated legally binding contract. By specifying the importance of every criterion, each party has the opportunity to clearly state which factors it considers important. Furthermore, it is possible to communicate the influence of these factors in the quality review which is conducted after the completion of the business interaction. This ensures a fair and transparent measurement of the QoS.

After its completion, the both parties review the business interaction according to the agreed quality criteria. The agent then calculates the correlation value between the expected and the actual business interaction outcome using the proposed Fuzzy Service Quality Review model. We use the three variables from the CCCI metrics as input for our QoS fuzzy inference engine; commitment to a criterion, clarity of a criterion and influence of a criterion. In the following, we will introduce each of these variables and explain how we integrate them into our QoS measurement model.

\section{A. Commitment to a Criterion}

The commitment to each criterion (service condition), to which the Trusted Agent and the Trusting Agent mutually agreed upon, before the business interaction, measures the actual degree of fulfilment of every specified criterion. For example, the Trusted Agent delivered a hatchback model instead of the requested sedan. This breach of the service contract is rated by the Trusting Agent as a 'partial delivery' of a 'very important' contract criterion and will, thus, influence the trustworthiness assessment of the Trusted Agent negatively. 
TABLE III

SEMANTICS \& DESCRIPTION OF THE COMMITMENT CRITERION

\begin{tabular}{|c|c|c|c|}
\hline $\begin{array}{l}\text { Semantics } \\
\text { (Deliver } \\
\text { the } \\
\text { service) }\end{array}$ & Description & $\begin{array}{c}\text { Values of } \\
\text { Commit }_{\text {criterion }} \\
\text { c }\end{array}$ & $\begin{array}{c}\text { Fuzzy } \\
\text { membership } \\
\text { function label }\end{array}$ \\
\hline $\begin{array}{l}\text { Nothing is } \\
\text { delivered }\end{array}$ & $\begin{array}{l}\text { The provider } \\
\text { (Trusted Agent) did } \\
\text { not fulfil any of the } \\
\text { commitments. }\end{array}$ & $x=0$ & 'nothing' \\
\hline $\begin{array}{l}\text { Minimally } \\
\text { delivered }\end{array}$ & $\begin{array}{l}\text { The provider only } \\
\text { delivered a little bit } \\
\text { of what was } \\
\text { committed. }\end{array}$ & $0<x \leq 1$ & 'minimal' \\
\hline $\begin{array}{l}\text { Partially } \\
\text { delivered }\end{array}$ & $\begin{array}{l}\text { The provider only } \\
\text { delivered half of } \\
\text { what was } \\
\text { committed. }\end{array}$ & $1<x \leq 2$ & 'partially' \\
\hline $\begin{array}{c}\text { Largely } \\
\text { Delivered }\end{array}$ & $\begin{array}{l}\text { The provider } \\
\text { delivered a large } \\
\text { portion of the } \\
\text { contract he } \\
\text { committed to. }\end{array}$ & $2<x \leq 3$ & 'largely' \\
\hline Delivered & $\begin{array}{c}\text { The provider's } \\
\text { service delivery is } \\
\text { satisfactory and all } \\
\text { commitments were } \\
\text { honoured. }\end{array}$ & $3<x \leq 4$ & 'satisfactory' \\
\hline $\begin{array}{c}\text { Fully } \\
\text { delivered }\end{array}$ & $\begin{array}{l}\text { The provider has } \\
\text { fully delivered on } \\
\text { the commitment. }\end{array}$ & $4<x \leq 5$ & 'fully' \\
\hline
\end{tabular}

Due to their fuzzy nature, the commitment labels and their semantics (Table ) are difficult to be measured by the agent. One solution to approach this problem would be the definition of value ranges for each commitment level as depicted in Table. However, this approach does not reflect the fuzzy nature of the commitment measurement process since it still enforces crisp boundaries between the seven levels. For this reason, we have chosen to model the commitment variable as a fuzzy variable as depicted in Figure 1.

The replacement of these value ranges by fuzzy membership functions improves those shortcomings, because the commitment of a criterion can belong to several fuzzy sets at the same time. This approach reflects the human thought process which uses labels of fuzzy sets rather than crisp numbers. Fuzzy logic allows a gradual transition from membership to non-membership functions. For example, a measured commitment value of 1.4 would be labelled 'partial commitment' when applied to a crisp rating system, but in a fuzzy system, it would belong to a degree of $40 \%$ to the 'partially' fuzzy set and $60 \%$ to the 'minimal' fuzzy set.

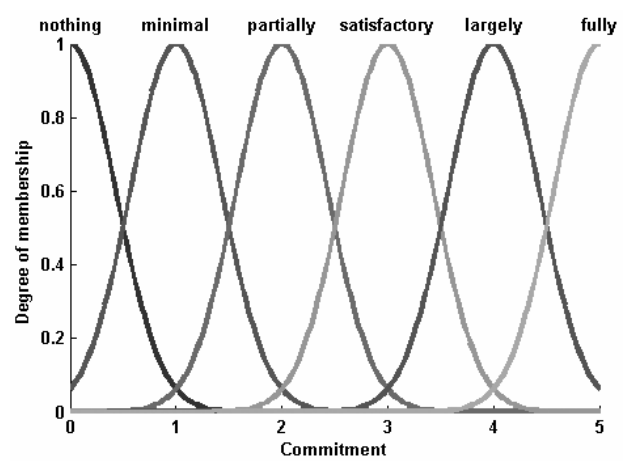

Figure 1. Fuzzy membership functions for commitment variable

\section{B. Clarity of a Criterion}

We also need to assess the clarity of each criterion (service condition). This provides a measure to establish if each criterion was clearly specified, commonly understood and mutually agreed to between the Trusting and the Trusted Agent. Due to the imprecise nature of the clarity variable, we model it as fuzzy variable with three fuzzy sets labelled 'low', 'medium', and 'high':

TABLE IV

SEMANTICS, DESCRIPTION, VALUE RANGES FOR CLARITY CRITERION

\begin{tabular}{|c|c|c|c|}
\hline Semantics & Description & $\begin{array}{c}\text { Values of } \\
\text { Clear criterion c }\end{array}$ & $\begin{array}{c}\text { Fuzzy } \\
\text { membership } \\
\text { function label }\end{array}$ \\
\hline not clear & $\begin{array}{c}\text { This criterion is } \\
\text { not clearly } \\
\text { defined }\end{array}$ & $0<\mathrm{x} \leq 2.5$ & 'low' \\
\hline $\begin{array}{c}\text { partially } \\
\text { clear }\end{array}$ & $\begin{array}{c}\text { Some Criteria are } \\
\text { not clear }\end{array}$ & $0<\mathrm{x} \leq 5$ & 'medium' \\
\hline very clear & $\begin{array}{c}\text { The criterion is } \\
\text { generally clear }\end{array}$ & $2.5<\mathrm{x} \leq 5$ & 'high' \\
\hline
\end{tabular}

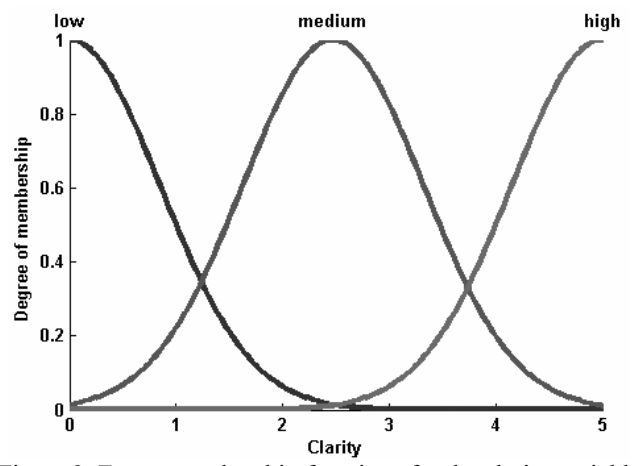

Figure 2. Fuzzy membership functions for the clarity variable

In our example, where the Trusted Agent delivered a hatchback model instead of a sedan, the Trusting Agent rates the clarity of the 'body type' criterion as 'high'. This is because the 'body type criterion' was clearly expressed, 
understood and agreed to by both parties in the service contract which was negotiated prior to the business interaction.

\section{Influence of a Criterion}

Furthermore, our model specifies the influence or importance of each criterion as an additional input variable. Prior to the business interaction, the influence value for each criterion is predefined by the agent owner during the definition of the quality criteria (see Table ). For example, the agent owner may find that a complete and timely delivery of the ordered goods or services is more important then the payment method. The Trusting Agent communicates this value for each criterion during the contract negotiation in order to make the QoS Review as transparent as possible.

Again, we model this value as a fuzzy variable which offers the agent owner the opportunity to express the influence of a criterion as a set of linguistic variables rather then crisp numbers:

TABLE V

SEVEN LEVELS FOR THE INFLUENCE CRITERION

\begin{tabular}{|c|c|c|c|}
\hline Semantics & Description & $\begin{array}{l}\text { Value of } \\
\text { Inf }_{\text {crite rion c }}\end{array}$ & $\begin{array}{c}\text { Fuzzy } \\
\text { membership } \\
\text { function } \\
\text { label }\end{array}$ \\
\hline unimportant & $\begin{array}{l}\text { This weight indicates } \\
\text { that the criterion is of } \\
\text { very little importance to } \\
\text { the agent owner. }\end{array}$ & $0<x \leq 2.5$ & 'low' \\
\hline $\begin{array}{l}\text { partially } \\
\text { important }\end{array}$ & $\begin{array}{l}\text { This weight indicates } \\
\text { that the criterion is of } \sim \\
50 \% \text { importance }\end{array}$ & $0<x \leq 5$ & 'medium' \\
\hline $\begin{array}{c}\text { very } \\
\text { important }\end{array}$ & $\begin{array}{l}\text { This weight indicates } \\
\text { that the criterion is } \\
\text { crucial for the Trusting } \\
\text { Agent and if the Trusted } \\
\text { Agent does not satisfy } \\
\text { this criterion, it may be } \\
\text { assigned a low QoS } \\
\text { value. }\end{array}$ & $2.5<x \leq 5$ & 'high' \\
\hline
\end{tabular}

Tables 1 and 2 demonstrate the usage of importance in our car purchasing example. Both agents define a set of criteria as part of their service contract. Each criterion in this contract is assigned an influence label to express its importance towards the measurement of the service quality or commitment to the business contract.

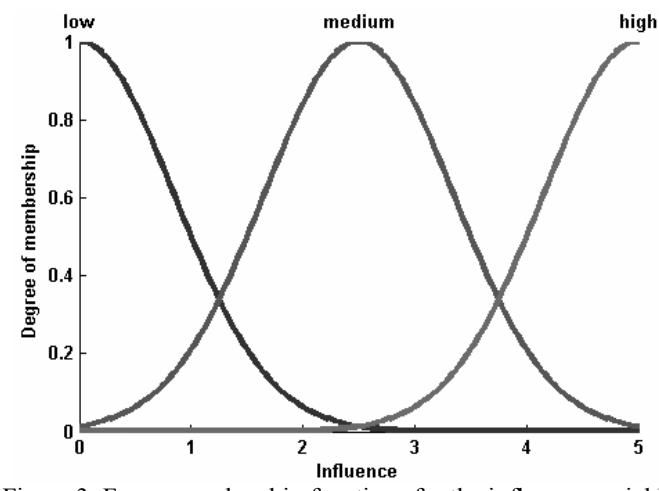

Figure 3. Fuzzy membership functions for the influence variable

In the following, we will provide details about the integration of the three fuzzy input variables into our fuzzy inference engine. This process will allow the measurement of service quality in SOAs.

\section{Fuzzy Service Quality Calculation}

Our approach of modelling the QoS in SOAs is based on a fuzzy system.

We model the above introduced metrics for commitment to a criterion, clarity of a criterion, and influence of a criterion as fuzzy variables, that is, each set is represented by fuzzy numbers and their associated linguistic variables. These fuzzy variables serve as input to our fuzzy QoS calculation

Figure 5. system as depicted in

It is, furthermore, necessary to define a fuzzy variable and its membership functions for the service quality output variable of the fuzzy QoS inference engine. Again, we use three fuzzy sets represented by their linguistic labels 'inadequate', 'sufficient', and 'excellent' to express the QoS variable (see Figure 4).

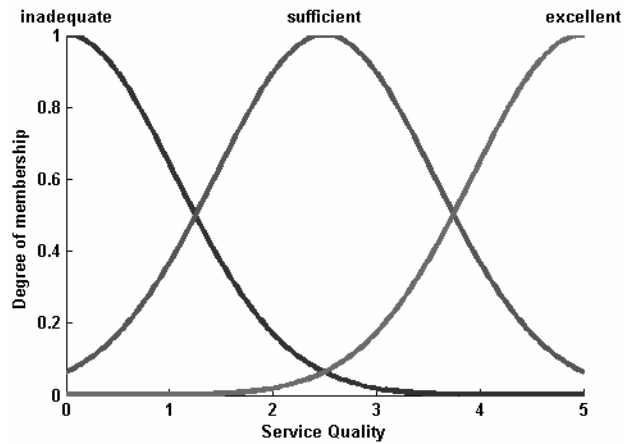

Figure 4. Fuzzy membership functions for the output 'service quality' variable 


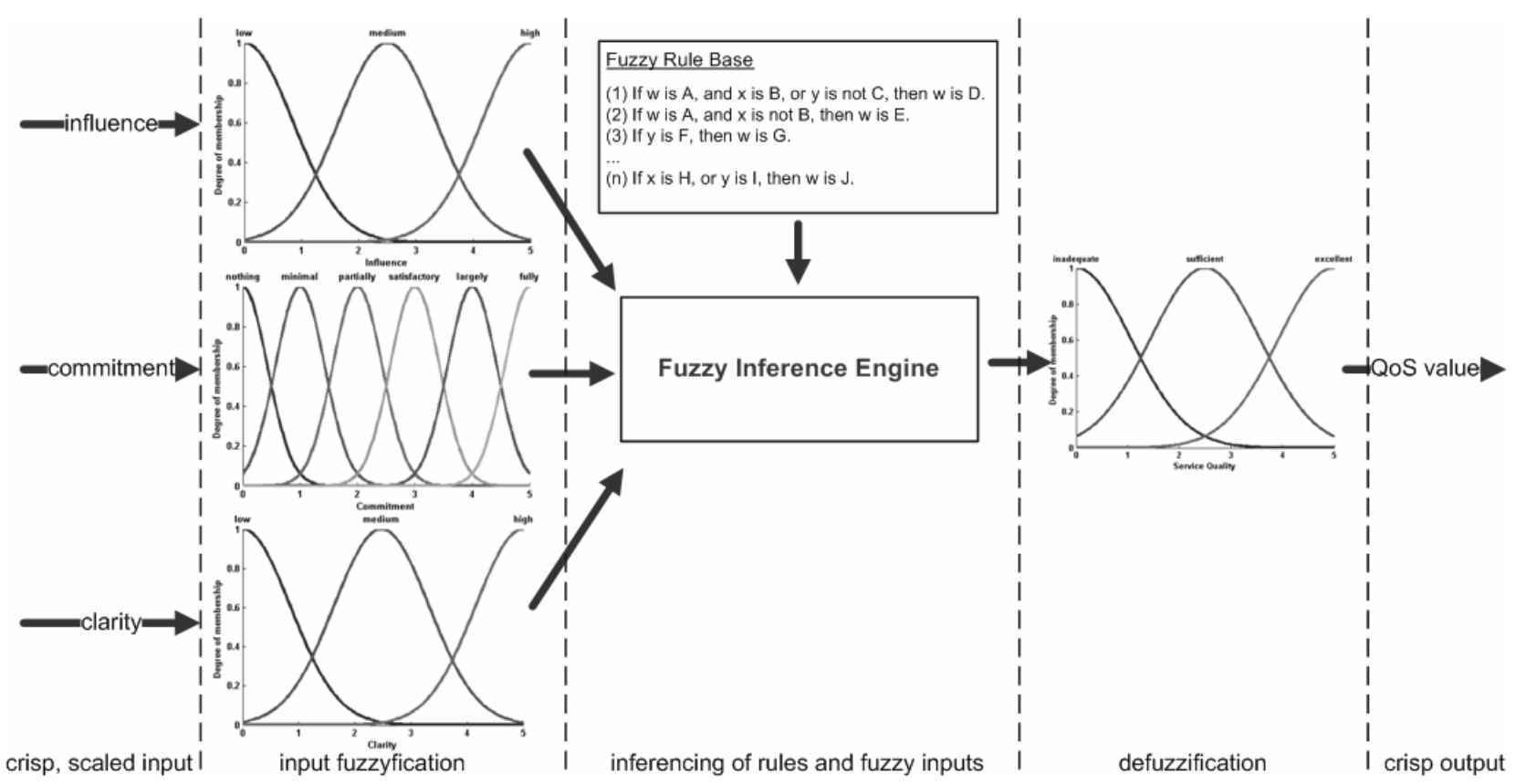

Figure 5. Fuzzy QoS calculation system architecture

Moreover, a context-specific set of inference rules, called fuzzy rule base has to be defined for the QoS fuzzy inference engine. These rules can be flexibly adjusted by the owner of the Trusting Agent to reflect his personal quality perception for the observed context. The fuzzy rule base, thus, represents the domain-specific knowledge of the agent owner. The rule base contains a maximum number of 54 rules $(3 * 3 * 6=54)$, based on the number of fuzzy sets specified within each input fuzzy variable for the QoS fuzzy engine. When allowing negations the maximum number will even rise to $432((3+3) *(3+3) *(6+6)=432)$ possible rules. However, the actual number of fuzzy rules is likely to be smaller than 54 (or 432 respectively) since many rules can be summarized. In fact, negations are often used to effectively decrease the number of rules since they allow the combination of fuzzy sets defined for a linguistic variable (see Figure 6). A decreased set of fuzzy rules will result in a reduction of calculations within the fuzzy engine and thus contribute to a better performance of the fuzzy system.

A fuzzy rule can be expressed as a combination of linguistic in the form of (several) fuzzy predicates in the antecedent as well as one predicate in the consequent block:

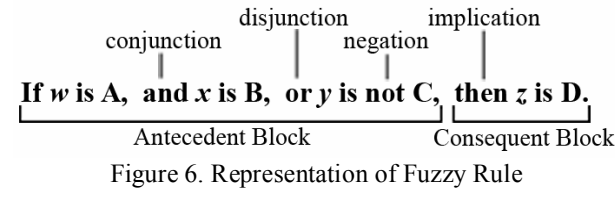

Conditions expressed as fuzzy rules can be combined in the form of conjunctions, disjunctions or combinations of both. Furthermore, fuzzy rules offer the possibility of using negations which adds another powerful tool for the creation of fuzzy rule bases [9].

As a last step, an appropriate defuzzification method needs to be selected, which, depending on the agent owner's personal preferences, could be the 'centre of area' method, the 'centre of maxima' method, or the 'mean of maxima' as discussed by Klir and Yuan [10]. Other popular defuzzification methods include the 'centre of gravity method', the 'mean of maxima' method, the 'first of maximum' method, or the 'last of maximum' method [11]. As a result of the defuzzification, the Trusting Agent receives the desired crisp service quality values for each quality criterion as specified in the contract of the reviewed business interaction. The computed QoS values for each quality criterion will then be weighted by their influence factor to compute an overall average QoS value.

The outcome of our model can then be used to adjust credibility records of third party agents which delivered opinions to update trustworthiness values in the business partner, or to communicate the trustworthiness of the business partner to interested peer agents $[8,12]$.

\section{EXAMPLE}

In this section, we demonstrate the proposed fuzzy service quality review model in an example classic book buying scenario. In this scenario, a service consumer agent is instructed by its owner to buy a specific book. In a first step, the agent obtains domain specific knowledge such as book types, delivery methods, payment methods, etc. In a second step, the agent selects a service provider (online bookshop) from a number of choices by applying our previously introduced model [7]. Then, the Trusting Agent negotiates 
the contract and service conditions with the Trusted Agent. These negotiations are bound to the pre-defined interaction policies which result from a preceding trustworthiness evaluation process [7]. The resulting contract also contains a set of service quality criteria as communicated and accepted by both parties. Table depicts a brief example of service quality criteria as defined by the Trusting Agent.

TABLE VI

QUALITY CRITERIA OF THE BOOK-BUYING EXAMPLE

\begin{tabular}{|c|l|c|}
\hline Criterion & \multicolumn{1}{|c|}{ Expected Delivery } & Influence \\
\hline Topic & $\begin{array}{l}\text { Trust and Reputation in Service Oriented } \\
\text { Architectures }\end{array}$ & 4.7 \\
\hline Type & Hard-cover book & 0.8 \\
\hline Delivery & Within 1 week & 1.3 \\
\hline Price & $<\$ 40$ & 2.8 \\
\hline Language & English, or Spanish & 1.7 \\
\hline $\begin{array}{c}\text { Publication } \\
\text { Date }\end{array}$ & $>2003$ & \\
\hline
\end{tabular}

After having carried out the business interaction (the product has been delivered and paid for), both parties apply our model to determine the commitment to the business contract which is defined as QoS. Since there is no conceptual difference between the QoS calculations of both parties, we only illustrate the Trusting Agent's activities to measure the QoS according to its quality criteria:

\section{A. Ranking of all quality criteria}

The Trusting Agent reviews the delivery of the business interaction and calculates the level of commitment and clarity as numerical values within a range of 0 to 5 :

TABLE VII

REVIEW OF COMMITMENT TO THE BUSINESS CONTRACT AND CLARITY OF THE CRITERION DESCRIPTIONS

\begin{tabular}{|c|l|c|c|}
\hline Criterion & \multicolumn{1}{|c|}{ Actual Delivery } & Commitment & $\begin{array}{c}\text { Clarit } \\
\mathbf{y}\end{array}$ \\
\hline Topic & $\begin{array}{l}\text { Trust, Reputation, and } \\
\text { Security: Theories and } \\
\text { Practice }\end{array}$ & 3.9 & 4.5 \\
\hline Type & Soft-cover book & 3.5 & 5 \\
\hline Delivery & 4 weeks & 1.5 & 4.1 \\
\hline Price & \$52.95 & 4 & 3.5 \\
\hline Language & English (brit.) & 3.7 & 1.8 \\
\hline $\begin{array}{c}\text { Publicatio } \\
\text { n Date }\end{array}$ & $\begin{array}{l}\text { Book published in 2003; } \\
\text { contents written in 2002 }\end{array}$ & 2.5 \\
\hline
\end{tabular}

B. Setup of Fuzzy Rule Base

The owner of the Trusting Agent pre-defined a number of fuzzy rules using the linguistic variables of the four fuzzy membership functions for influence, commitment, clarity and service quality. Table depicts an extract of this rule base:

TABLE IIX

EXTRACT OF FUZZY RULE BASE FOR BOOK-BUYING EXAMPLE

\begin{tabular}{|l|l|l|l|}
\hline Influence (A) & Commitment (B) & Clarity (C) & $\begin{array}{l}\text { Service Quality } \\
(\mathbf{Q o S})\end{array}$ \\
\hline If A is not high & and B is partially & and C is not high & $\begin{array}{l}\text { then QoS is } \\
\text { sufficient }\end{array}$ \\
\hline If A is low & $\begin{array}{l}\text { and B is not } \\
\text { nothing }\end{array}$ & and C is low & $\begin{array}{l}\text { then QoS is } \\
\text { sufficient }\end{array}$ \\
\hline & if B is fully & & $\begin{array}{l}\text { then QoS is } \\
\text { excellent }\end{array}$ \\
\hline If A is low & If B is nothing & & $\begin{array}{l}\text { then QoS is } \\
\text { inadequate }\end{array}$ \\
\hline
\end{tabular}

\section{Inference of Fuzzy Input Variables and Fuzzy Rule Base}

Using the numerical values of the influence, commitment and clarity variables (see Table and Table ) for its fuzzy engine as input data, as well as the fuzzy rule base (see Table ), the Trusting Agent can now calculate the service quality value for each criterion by inferring the fuzzified input variables with the fuzzy rule base. A graphical demonstration of this inference procedure for the 'language' criterion is depicted in Figure 7:

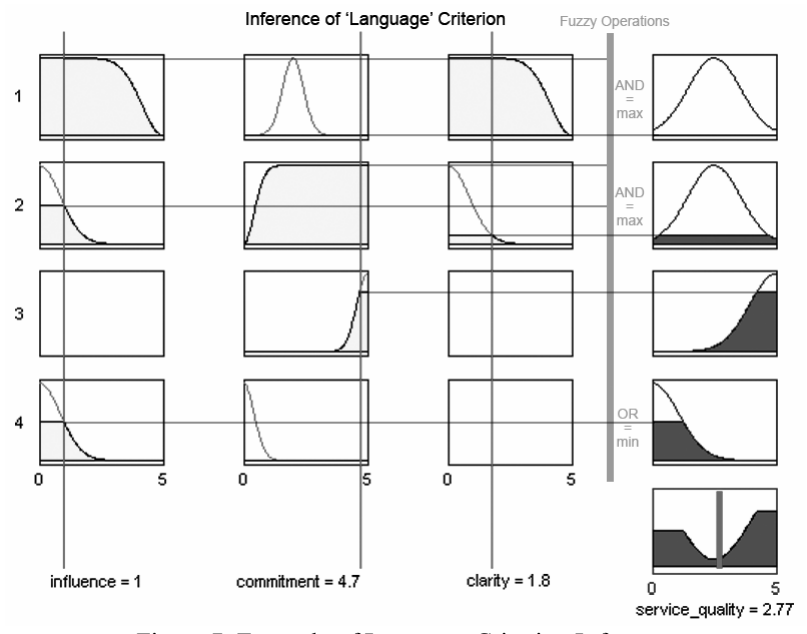

Figure 7. Example of Language Criterion Inference

This example demonstrates the superior characteristics of fuzzy set theory over interval partitions based on the classical set theory. For example, a clarity input value of 1.8 would be labelled as 'medium' if this crisp set was defined as:

$$
\text { medium }=\left\{a_{i} \mid 1 . \overline{6} \leq i \leq 3 . \overline{3}\right\}
$$

However, humans don't measure clarity within crisp sets, but rather use gradual transitions between the membership and non-membership of a value to a specific set. One could argue that it is possible to split the universe of the medium set into several subsets and, thus, reduce the granularity and increase the precision of the modelled variable (clarity), but 
this would require a significant increase in the number of policies or rules which define the model behaviour for each subset. The usage of fuzzy sets solves this problem and allows the system owner to decide how many rules are necessary to represent its knowledge without significantly compromising the desired precision of the model.

As a defuzzification method in this example we have chosen the 'centre of gravity' method as discussed in [11]. The fuzzy inference and defuzzification process is repeated for all quality criteria as defined in Table :

TABLE IX

DEFUZZIFICATION RESULTS FOR ALL CRITERIA

\begin{tabular}{|c|c|c|c|c|}
\hline Criterion & Influence & Commitment & $\begin{array}{c}\text { Clarit } \\
\mathbf{y}\end{array}$ & QoS \\
\hline Topic & 4.7 & 3.9 & 4.5 & 3.43 \\
\hline Type & 3 & 3.5 & 5 & 2.50 \\
\hline Delivery & 1.3 & 1.5 & 4.1 & 2.33 \\
\hline Price & 2.8 & 4 & 3.5 & 3.45 \\
\hline Language & 1 & 4.7 & 1.8 & 2.77 \\
\hline $\begin{array}{c}\text { Publication } \\
\text { Date }\end{array}$ & 1.7 & 3 & 2.5 & 2.03 \\
\hline
\end{tabular}

\section{Calculation of Overall QoS Value}

As a last step the Trusting Agent simply calculates an average value over all QoS values for each quality criterion. The resulting value represents the overall value for the quality of service of our book-buying example:

Overall QoS Value

$=\frac{3.43+2.50+2.33+3.45+2.77+2.03}{6}$

$=\underline{\underline{2.75}}$

This final QoS value can then be used to adjust credibility records of third party agents which delivered opinions, to update trustworthiness values in the business partner, or to communicate the trustworthiness of the business partner to interested peer agents.

\section{RELATED WORK}

In this section, we briefly discuss current research approaches for Quality of Service (QoS) measurement approaches.

In an environment, where an autonomous agent must choose between several discovered services without manual user intervention, the $\mathrm{QoS}$ is a decisive factor. The adherence to mutually agreed QoS criteria between autonomous agents in distributed environments can only be determined after the actual business interaction has been completed. Current literature mostly offers models where the QoS is determined through a fixed set of criteria. These criteria include transaction duration, reliability, security and availability of the services provided [13] among others. The measurement of service quality for services offered in the SOA has grown quite popular over the last years and is mostly based on a fixed set of functional factors.

For example, Zeng et al. propose a quality measurement framework for the selection of Web services in SOAs [14]. They analyse attributes such as price and reputation to calculate a composite QoS rating for discovered services. However, they measured the QoS of services offered in the SOA based on pre-defined factors which are generally applicable to all services offered. Their measurements do not consider criteria related to the fulfilment of individually negotiated service contracts. For example, the quality of a delivered product may significantly differ from its description in the service contract, such as if a book was specified as hard-cover book but was actually delivered as soft-cover version. Currently, there is little work published on service quality measurement for such context and contract-specific criteria as negotiated in service level agreements and contracts.

Maximilien and Singh propose a QoS measurement approach for agent-based trust calculation [15-17]. They suggest a model and an ontology which enables agents to exchange their QoS ratings. However, their approach assumes that exchanged opinions among agents are mostly truthful and, thus, untruthful opinions have no significant impact on exchanged opinions. Furthermore, the exchanged QoS ratings are based on user-defined criteria. The manual definition of QoS criteria requires high user involvement. This manual selection of attributes used for QoS measurement contradicts the autonomic nature of intelligent agents.

Our work concentrates on the measurement of contract fulfilment based on context and contract-specific criteria. Furthermore, we believe that the measurement of the QoS must be performed by the involved parties (consumer and provider) directly, rather than a third party [18] or a middleware layer $[19,20]$. Only a solution which uses decentralized measurement of non-functional QoS criteria provides sufficient scalability for the demands of autonomous agents operating in distributed e-commerce environments.

\section{CONClusion \& Future Work}

As Service Oriented Architectures become increasingly popular and are being put into practice in more and more distributed enterprise businesses, we need to introduce methodologies for the measurement of critical factors such as trust, credibility and quality of service. Only their measurement will enable SOAs to reach their full potential in dynamic and collaborative e-business scenarios.

An autonomous agent requires a comprehensive mechanism to determine the quality of a completed business interaction in a Service Oriented Environment. This mechanism must allow the agent to measure a set of context or domain specific criteria in an objective way. The 
measurement of criteria whose value ranges are commonly expressed as linguistic terms rather then crisp numbers represents an exceptional challenge for computing science.

This paper extended our previous work on trust evaluation and credibility development in distributed systems [7, 8] and proposed a fuzzy service quality review model for autonomous agents which act on behalf of their owners in Service Oriented Environments. We have taken advantage of fuzzy logic capabilities to deal with uncertainty and the tolerance of imprecise data inputs. We measure the commitment, clarity and influence of user-defined criteria as specified in the service contract. Such criteria are often used to express decisive factors for the evaluation of the quality of a service or product during human interaction.

In future work, we will validate our model in a P2P environment to assess the framework accuracy on a longterm basis. Eventually, our model can be incorporated into existing e-commerce markets as a key building block where autonomous agents interact by imitating human social behaviour.

\section{REFERENCES}

[1] DMReview, "DMReview Glossary," vol. 2005.

[2] E. Chang, F. Hussain, and T. Dillon, Trust and Reputation for Serviceoriented Environments, vol. 1: John Wiley \& Sons, 2005.

[3] Farookh Hussain, Elizabeth Chang, and Tharam Dillon, "Trustworthiness and CCCI Metrics for Assigning Trustworthiness in P2P Communication.," International Journal of Computer Systems Science and Engineering, vol. 19, pp. 95-112, 2004.

[4] L. A. Zadeh, "The concept of a linguistic variable and its application to approximate reasoning," Information Sciences, vol. 8, pp. 199-249, 1975.

[5] P. Bonissone, "A fuzzy sets based linguistic approach: Theory and applications," presented at 12th conference on Winter, simulation, 1980.

[6] L. A. Zadeh, "Fuzzy sets," in Fuzzy sets, fuzzy logic, and fuzzy systems: selected papers by Lotfi A. Zadeh: World Scientific Publishing Co., Inc., 1996, pp. 19-34.

[7] S. Schmidt, R. Steele, T. Dillon, and E. Chang, "Building a Fuzzy Trust Network in Unsupervised Multi-agent Environments," in Lecture Notes in Computer Science, 2005, pp. 816-825.

[8] S. Schmidt, R. Steele, T. Dillon, and E. Chang, "Applying a Fuzzy Trust Model to E-Commerce Systems," in Lecture Notes in Computer Science, 2005, pp. 318-329.

[9] K. H. Lee, First Course on Fuzzy Theory and Applications. Heidelberg: Springer, 2005.

[10] G. J. Klir and B. Yuan, Fuzzy Sets and Fuzzy Logic: Theory and Applications: Prentice Hall PTR, 1995.

[11] L. Werner Van and E. K. Etienne, "Defuzzification: criteria and classification," Fuzzy Sets Syst., vol. 108, pp. 159-178, 1999.

[12] Stefan Schmidt, Robert Steele, and T. Dillon, "Fuzzy Trust Evaluation and Credibility Development in Multi-Agent Systems," accepted to Apllied Soft Computing Journal, Elsevier, 2006.

[13] J. O'Sullivan, D. Edmond, and A. T. Hofstede, "What's in a Service?," Distrib. Parallel Databases, vol. 12, pp. 117-133, 2002.

[14] L. Zeng, B. Benatallah, M. Dumas, J. Kalagnanam, and Q. Z. Sheng, "Quality driven web services composition," in Proceedings of the 12th international conference on World Wide Web. Budapest, Hungary: ACM Press, 2003.

[15] E. M. Maximilien and M. P. Singh, "Agent-based trust model involving multiple qualities," in Proceedings of the fourth international joint conference on Autonomous agents and multiagent systems. The Netherlands: ACM Press, 2005.

[16] E. M. Maximilien and M. P. Singh, "A framework and ontology for dynamic Web services selection," Internet Computing, IEEE, vol. 8, pp 84-93, 2004.

[17] E. M. Maximilien and M. P. Singh, "Toward autonomic web services trust and selection," in Proceedings of the 2nd international conference on Service oriented computing. New York, NY, USA: ACM Press, 2004

[18] S. Ran, "A model for web services discovery with QoS," SIGecom Exch., vol. 4, pp. 1-10, 2003.

[19] E. Wohlstadter, S. Tai, T. Mikalsen, I. Rouvellou, and P. Devanbu, "GlueQoS: Middleware to Sweeten Quality-of-Service Policy Interactions," in 26th International Conference on Software Engineering. Scottland, UK, 2004.

[20] L. Zeng, B. Benatallah, A. H. H. Ngu, M. Dumas, J. Kalagnanam, and H. Chang, "QoS-aware middleware for Web services composition," Software Engineering, IEEE Transactions on, vol. 30, pp. 311-327, 2004. 